\title{
PHC re-engineering may relieve overburdened tertiary hospitals in South Africa
}

\author{
Morongwa Caroline Mohapi, Debashis Basu
}

Under the National Health Insurance, a hospital is expected to provide service to patients based on its category. However, in reality the tertiary hospitals offer every level of care, resulting in poor quality of care and over-expenditure. The Polokwane/Mankweng Hospital Complex is a provincial tertiary hospital that delivers tertiary care as well as dealing with some secondary and primary care cases. This study evaluated the hospital casualty department in the Polokwane/Mankweng Hospital Complex. A sample of 250 patients' records was selected by simple random sampling from a cohort of 14113 patients who attended the Polokwane Hospital Casualty Department during the 1-year study period. Most patients were admitted in the casualty department as a result of injuries $(25 \%)$. Only $20 \%(N=51)$ of the patients were referred from other health facilities. Half of the patients could have been managed at a regional or district hospital. The overall expenditure for the casualty unit during the 1-year study period was R10 321401.42 and the combined unit cost was estimated at R731.34 per single emergency care patient excluding the capital costs.

Referral systems must be strengthened to manage patients at regional and district level to reduce the burden on the Polokwane/ Mankweng Hospital Complex. It is hoped that the Primary Health Care (PHC) Re-engineering Policy will address this by strengthening the referral system in PHC facilities.

S Afr Med J 2012;102:79-80

\section{Background}

Under the National Health Insurance, a hospital is expected to provide service to patients based on its category, e.g. tertiary hospitals should provide specialist level services (provided by regional hospitals, subspecialty care, and critical care under the supervision of a specialist) and should receive referrals from regional hospitals not limited to provincial boundaries. ${ }^{1}$ Therefore regional and district hospitals should be able to cater for other levels of care. In reality, the tertiary hospitals offer all levels of care, resulting in poor quality of care and over-expenditure. ${ }^{2}$ Providing basic services in a tertiary hospital casualty department creates problems for the casualty department and for the entire healthcare system. ${ }^{3}$

The Polokwane/Mankweng Hospital Complex (comprising two hospitals situated $30 \mathrm{~km}$ apart in Polokwane and Turfloop) is a provincial tertiary hospital ${ }^{1}$ that delivers tertiary care and deals with some secondary and primary care cases. This complex will be home to the new medical school ${ }^{4}$ and is one of the six flagship hospitals to be built in the next few years. ${ }^{5}$ The province has five regional hospitals that refer healthcare users to the complex, and 30 district hospitals, most of which, because of the proximity between them and regional hospitals, prefer to transfer patients directly to the complex. During 2006/2007, the number of casualty consultations at the complex reached a total of $41768 .{ }^{6}$ This huge patient load affects the quality of care in the hospital casualty department and increases their staff workload. This problem needed to be quantified and addressed before the hospital complex is converted into a fully fledged tertiary hospital.

School of Public Health, University of the Witwatersrand, Johannesburg Morongwa Caroline Mohapi, MPH Hospital Management student

Department of Community Health, Charlotte Maxeke Johannesburg Academic Hospital and University of the Witwatersrand

Debashis Basu, MSc (Med), MPH, MBA, MMed, FCPHM (SA), PhD (Med)

\section{Aim}

We aimed to evaluate the hospital casualty department in the Polokwane/Mankweng Hospital Complex in terms of caseload, influencing factors and implications on resource utilisation.

\section{Methods}

A cross-sectional study design was used. A retrospective record review between April 2008 and March 2009 extracted information from various sources of hospital information systems. A sample of 250 patient records was selected by simple random sampling from a cohort of 14113 patients who attended the Polokwane Hospital Casualty Department. Data were collected on variables relevant to the functioning of and resource utilisation in the casualty unit of this complex. The project was approved by the University of the Witwatersrand Human Research Ethics Committee (Medical).

\section{Results}

Clinical profile. Approximately half of the patients were from the age group 25 - 44 years. More male patients were referred than female. Patients with medical aid bypassed the referral system more often than those without medical aid.

Most patients were admitted in the casualty department as a result of injuries (25\%), a similar finding to a study in Mpumalanga. ${ }^{7}$ Other common conditions were maternal conditions (7\%) and cardiovascular diseases (6\%) (Fig. 1).

Referral. Only $20 \%(N=51)$ of the patients were referred from other health facilities; $45 \%(N=112)$ of the patients were discharged, and $5 \%(N=13)$ were transferred back to the referring hospital, which implied that they could be managed at a regional or district hospital. Similar trends of non-emergency patients visiting the emergency department occur in other parts of the world. ${ }^{8-10}$

Almost half of the patients arrived after hours (18h01 - 06h00), indicating the need for after-hours services in primary healthcare (PHC) facilities as well as district and regional hospitals.

Cost. The overall expenditure for the hospital casualty department during this period was R10 321401.42 (R954 168.45 for pharmacy products, R177 261.16 for other consumables and R 1866233.25 for laboratory tests). Personnel expenditure was R7 323804 . 


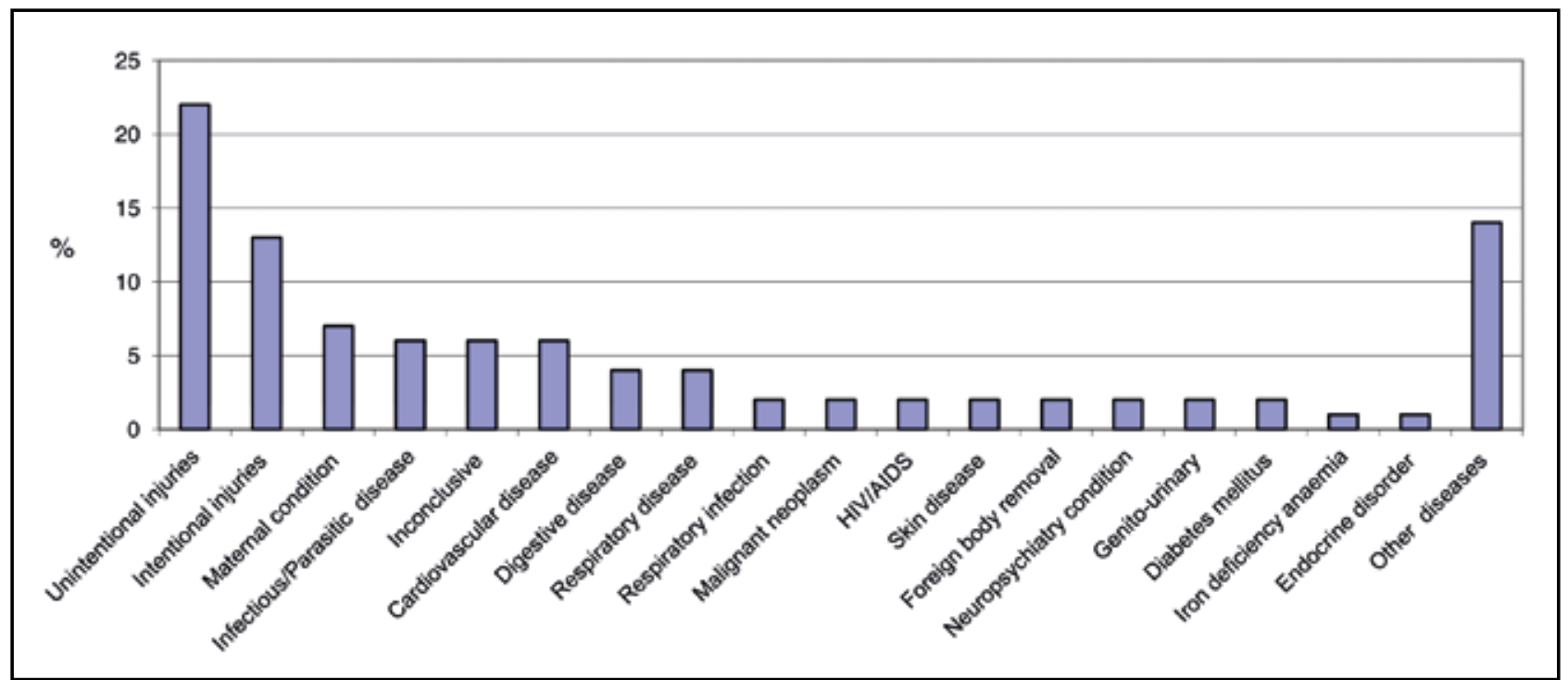

Fig. 1. Categories of diseases or injuries seen at casualty.

Unit personnel cost per patient was estimated at R518.94 (70.96\% of total recurrent cost), while the unit costs for the pharmaceuticals, stores and laboratory tests were R67.23 (9.24\%), R12.56 (1.72\%) and R132.24 (18.08\%) per patient, respectively. Overall the combined unit cost was estimated at R731.34 per single emergency care patient excluding the capital costs.

\section{Conclusion}

Appropriate measures to interface between tertiary institutions and other levels of care to provide seamless healthcare at affordable costs for patients and the system seem inevitable. Without these, Polokwane Hospital, and in particular the casualty department, would be used inappropriately by patients as an alternative to other providers of healthcare, namely provincial and district hospitals and other PHC units. This would be at the expense of other patients truly requiring care at a tertiary hospital. The $\mathrm{PHC}$ re-engineering ${ }^{11}$ policy will, it is hoped, address this by strengthening the referral system in PHC facilities.

\section{References}

1. National Health Act (Act 61 of 2003): Regulations: Categories of hospitals. 2011. Government Gazette, 554(34521).

2. Tanner M. Strengthening district health systems. Bull World Health Organ 2005;83(6):403.

3. Kobusingye OC, Hyder AA, Bishai D, Hicks ED, Mock C, Joshipura M. Emergency medical systems in low- and middle-income countries: recommendations for action. Bull World Health Organ 2005;83(8):626-631.

4. Department of Health. Human Resources for Health South Africa 2030. Pretoria: Department of Health, 2011 . 5. National Health Act (Act 61 of 2003): Policy on National Health Insurance. Government Gazette,

554(34523).

. Limpopo Department of Health and Social Development. Final Limpopo DHSA 2008/09-11 Annual Performance Plan - Vote 7. 2009. www.dhsa.limpopo.gov.za.../final\%20DHSD\%202008-9-11\%20 APP\%20Vote\%207 (accessed 7 June 2009).

7. Nkombua L. The practice of medicine at a district hospital emergency room: Middleburg Hospital, Mpumalanga province. South African Family Practice 2008;50(1):65.

8. Bezzina A, Smith P, Cromwell D, Eagar K. Primary care patients in the emergency department: who are they? A review of the definition of the 'primary care patient' in the emergency department. Emergency Medicine Australasia 2005;17(5/6):472-479.

9. Gentile S, Vignally P, Durand A, Gainotti S, Sambuc R, Gerbeaux P. Nonurgent patients in the emergency department? A French formula to prevent misuse. BMC Health Serv Res 2010;15;10:66. 10. McGuigan T, Watson P. Non-urgent attendance at emergency departments. Emergency Nurse 2010;18(6):34-38.

11. Department of Health. Re-engineering Primary Health Care in South Africa: Discussion document. Pretoria: Department of Health, 2010.

Accepted 24 November 2011. 